\title{
Cycling Performance in Short-term Efforts: Laboratory and Field- Based Data in XCO Athletes
}

\section{(요 $\odot \Theta$}

\author{
Authors \\ Patrick Schneeweiss ${ }^{1}$, Philipp Schellhorn ${ }^{1}$, Daniel Haigis ${ }^{1}$, Andreas Niess ${ }^{1}$, Peter Martus ${ }^{2}$, Inga Krauss ${ }^{1}$
}

\begin{abstract}
Affiliations
1 Medical Clinic, Department of Sports Medicine, University of Tübingen, Tübingen, Germany

2 Institute for Clinical Epidemiology and Applied Biometry, University of Tübingen, Tübingen, Germany
\end{abstract}

Keywords

power output, cadence, mountain bike, off-road cycling, pedaling frequency

$\begin{array}{ll}\text { received } & 07.10 .2019 \\ \text { revised } & 16.01 .2020 \\ \text { accepted } & 20.01 .2020\end{array}$

Bibliography

DOI https://doi.org/10.1055/a-1101-5750

Sports Medicine International Open 2020; 4: E19-E26

(c) Georg Thieme Verlag KG Stuttgart · New York

ISSN 2367-1890

\section{Correspondence}

Patrick Schneeweiss

Medical Clinic, Department of Sports Medicine, University of Tübingen

Hoppe-Seyler-Strasse 6

72076 Tübingen

Germany

Tel.: + 497071/2986496

patrick.schneeweiss@med.uni-tuebingen.de $\circledast$ Supporting Information for this article is available online at http://www.thieme-connect.de/products.

\begin{abstract}
Mountain bike cross-country Olympic has an intermittent performance profile, underlining the importance of short-term but high cycling power output. Previous findings indicate that power output during sprint tests differs between laboratory and field-based conditions and that cycling cadence rises with increasing workload. The aim was therefore to examine power output and cadence in short-term efforts under laboratory and field conditions. Twenty-three competitive athletes $(17.9 \pm 3.7$ years) performed a laboratory power profile test and a simulated race within one week. Power output and cadence during the power profile test were compared to corresponding short-term efforts during the race over durations of $10-300 \mathrm{~s}\left(\mathrm{TT}_{10-300}\right)$. Differences were $\mathrm{TT}_{10}+8 \%, \mathrm{TT}_{30}+7 \%, \mathrm{TT}_{60}-15 \%$ and $\mathrm{TT}_{300}-12 \%$ for power output and $+10 \%,+8 \%,+19 \%,+21 \%$ for cadence respectively. Compared to the race, we found higher power output during the power profile test for the shorter efforts but lower for $\mathrm{TT}_{60}$ and $\mathrm{TT}_{300}$. Confirming previous results, cadence was higher during the power profile test compared to the respective intervals of the race and increased with increasing workload or shorter time trial duration. Future research should take into account that compared to the field, a higher cadence is used in laboratory settings to produce similar power output.
\end{abstract}

\section{Introduction}

Mountain bike cross-country Olympic (XCO) is one of four disciplines of mountain biking (MTB). XCO races get started with a mass start and are held over undulating circuits with technical descents, forest roads, rocky paths, and obstacles. Elite athletes have to finish 4-7 laps on a course, which is 4-6 km long, leading to race durations from 80 up to $100 \mathrm{~min}$ [1]. This cycling event is regarded as a highly intensive intermittent activity due to its large number of alternating climbs and descents [2-5]. Because the race duration was shortened several times in recent years and the technical sections of the course continuously increased, the race profiles became physiologically more irregular and technically more demanding with regard to the athletes' requirements $[1,6]$. Today, training in mountain biking is focused on performance-based training levels and modified high-intensity training zones to address short- and medium-term high-load events, especially in XCO racing $[6,7]$.

These physiological requirements should be reflected in performance tests to improve the training prescription and to evaluate the effectiveness of the training, especially in competitive athletes $[8,9]$. Laboratory tests should assess a cyclist's maximum capacity to produce power over durations that are typically encountered during races. Consequently, findings of specific laboratory tests can 
be directly compared to the cyclists' performance during competition. However, laboratory conditions are standardised. In contrast, competitions can take place in a variety of environmental and tactical conditions, and during the competition it is not always required that a given cyclist provides maximal efforts across the range of durations assessed in the laboratory test. Thus, the power produced during laboratory tests may differ from those produced in competition. This may have led to studies using sprints or timetrails in field-based conditions instead of real competitions when comparing cyclists' performance with data achieved during laboratory tests. Some earlier studies have concluded that, at least for longer time trails (lasting more than $20 \mathrm{~min}$ ), the average power produced during laboratory tests is not different to the cycling power output (PO) produced during real cycling time trials in the field, and is therefore a valid predictor [10-12]. However, some recent studies investigating the difference in cycling performance during sprint tests under laboratory and field-based conditions led to inconsistent results. Quod et al. [13] compared laboratory PO and cycling cadence (CAD) data with road race data of ten male cyclists and found no maximum mean PO differences for durations of 60-600 s. In contrast, the authors reported normalized differences of $3-9 \%$ for 5,15 , and 30 s duration sprints. Although PO achieved in the lab and field were at least similar in this study, the self-selected CAD to produce these efforts was remarkably higher in the lab (7-27 rpm). Gardner et al. [14] reported neither PO nor CAD differences during laboratory $6 \mathrm{~s}$ "all-out" sprints and $65 \mathrm{~m}$ sprints in seven elite track cyclists, and therefore concluded that velodrome performance can be accurately modelled using laboratory-based data. Bertucci et al. [15] reported both higher ( +6\%, standing position) and lower ( $-4 \%$, seated) PO records during field compared to stationary ergometer sprinting. Their results indicate that laterally oscillating the bike can improve performance during short sprint tests in a standing position because in this position a higher perpendicular force can be applied to the crank and thus a higher propulsive force is generated.

Whether cycling in the laboratory, under field conditions, or in competition, the highest $\mathrm{PO}$ is reached with optimal values of force and pedalling cadence [16-19]. In order to achieve a certain PO, the athlete can choose either a high CAD and transfer a low force to the pedals, or vice versa. For many decades, researchers have been trying to find the optimal CAD in cycling. Most of them examined the effect of CAD on the economy, but other measures are also taken. The term "optimal cadence" has quite different meanings depending on whether it refers to the most economical, maximum power producing, less tiring, or most comfortable CAD $[16,17,20]$. Several factors, including age, $\mathrm{PO}$, and gradient, have been shown to affect the choice of CAD in cycling to some extent [21]. It is long known that there is an optimal contraction rate for muscle contractions [22]. Whether this leads to a given "optimal cadence" at various workloads during cycling is not obvious because the efficiency of the entire muscle could change with different force-speed ratios [23].

A number of studies that examined the relationship between CAD and performance or cycling economy suggest that the most economical CAD in cycling rises with increasing workload $[18,20,21,23-26]$. However, they refer almost exclusively to low, moderate, or submaximal cycling intensities, and performance does not only depend on cycling economy but also in a large part on the maximum energy turnover rate. Therefore, the most economical CAD is not necessarily the optimal one, especially for shortterm intervals as they frequently occur at XCO. Summarizing the findings through today, it can be assumed that a higher CAD (100120 rpm) improves sprint cycling performance, because muscle force and neuromuscular fatigue are reduced and $\mathrm{PO}$ is maximised [16], whereas the most economical CAD for submaximal workloads seems to be much lower ( $~ 80 \mathrm{rpm})$ and rises with increasing workload. To the best of our knowledge, there are no comparable studies at XCO competitions.

Based on the aforementioned findings, the present study aimed to compare PO and CAD during short-term time trials (TT; 10300 s) under laboratory and field conditions. In addition, we aimed to describe the relationship between PO and CAD when cycling at higher intensities. We hypothesised that i) PO during laboratory and field conditions is not significantly different, ii) CAD is higher in the laboratory setting, and iii) CAD increases with increasing workload in both laboratory and field conditions.

\section{Materials and Methods}

\section{Participants}

The study meets the ethical standards required [27]. Ethical approval was received from the local ethics committee number 472/2016BO1 and the study was registered in the national database number PR020160800134. Suitable participants were recruited via trainers, clubs, and personal contacts within the MTB community. Following a telephone screening, 30 XCO athletes were invited to an initial visit $>$ Fig. 1S. All participants signed an informed consent and were examined for medical contraindications to exercise by a medical doctor.

\section{Procedures}

Following the medical examination and anthropometric measures, all athletes performed a mountain bike-specific performance test (MTB-PT, - Fig. 1), similar to the one elaborated by Ahrend et al. [28] and recently examined in XCO athletes [9]. The authors reported that the MTB-PT explained $57 \%\left(\mathrm{TT}_{10}\right), 72 \%\left(\mathrm{TT}_{30}\right), 70 \%\left(\mathrm{TT}_{60}\right)$, and $74 \%\left(\mathrm{TT}_{300}\right)$ of the variance in $\mathrm{PO}$ during an XCO race [9].

Within one week, this laboratory test was followed by a simulated XCO race (Race). The athletes were advised to avoid strenuous physical activity, alcohol, and other drugs for at least $24 \mathrm{~h}$ prior to the MTB-PT and the Race and to follow their usual preparation for competitions.

\section{Power Profile Test (PPT)}

On a Cyclus2 ergometer (RBM elektronik-automation, Leipzig, Germany) a standard MTB frame was adjusted to the specific demands of the athlete (seat post, stem slope, handlebar, and pedal kit). Additionally, the MTB frame was equipped with an SRM training system which consists of a power meter (instrumented crank) and a power control (PC8; data logger and on-board data display; Schoberer Rad Messtechnik, Welldorf, Germany). Because of its high validity, reliability and sensitivity, the SRM is considered the gold standard in mobile power meters [29, 30]. PO, CAD, and heart 


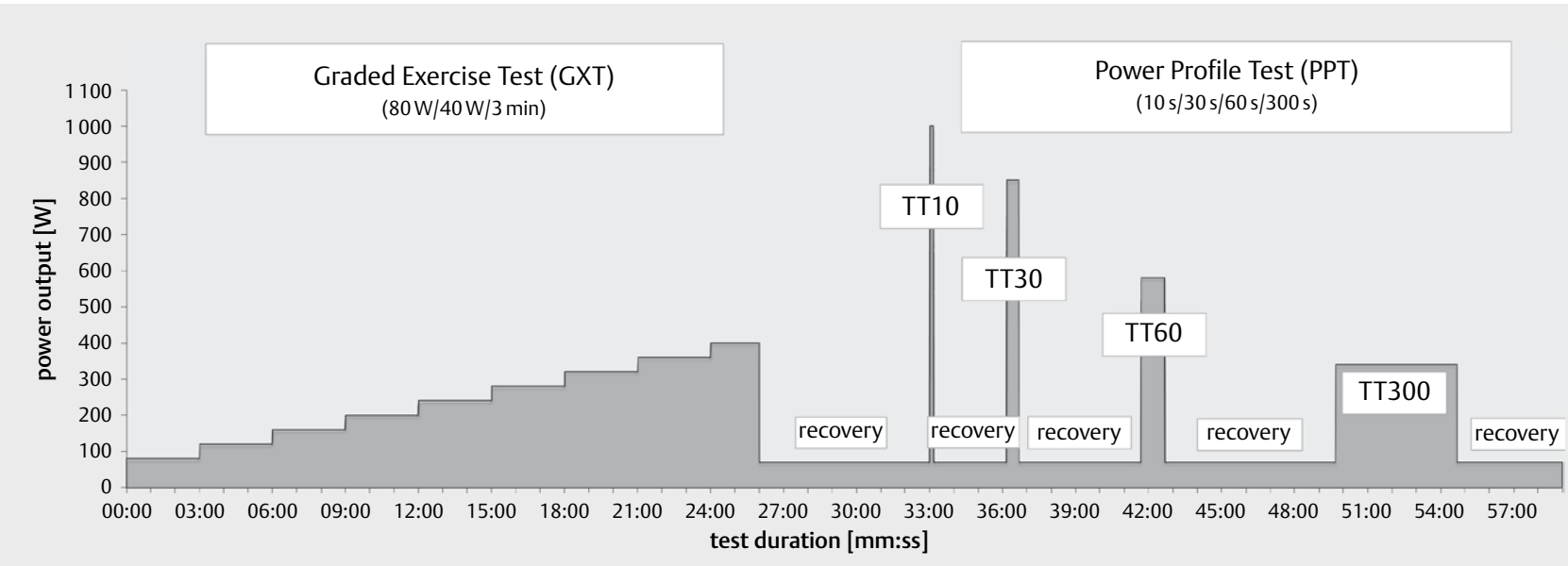

- Fig. 1 MTB-specific performance test (MTB-PT); $\mathrm{TT}_{10-300}$ : time trials lasting 10-300 s (sprint/maximal effort); recovery: period of recovery at $1.2 \mathrm{~W}^{*} \mathrm{~kg}^{-1}$ body mass

rate of the athletes were continuously recorded by the PC8 via $\mathrm{ANT}^{+}$at $1 \mathrm{~Hz}$. With the Cyclus 2 ergometer's elastic suspension, lateral oscillations are possible, so the laboratory test feels more like riding in a real MTB.

After a graded exercise test (GXT), which started at 80 watts and was increased by 40 watts every 3 min until subjective exhaustion, athletes continued pedalling for $7 \mathrm{~min}$ (recovery period) and then proceeded with the Power Profile Test (PPT) that comprised: i) a 10-s all-out sprint $\left(\mathrm{TT}_{10}\right)$; ii) a 3-min recovery period followed by a 30 -s all-out sprint ( $\mathrm{TT}_{30}$ ); $\mathrm{iii}$ ) a 5 -min recovery period followed by a 60 -s maximal effort ( $\left.\mathrm{TT}_{60}\right)$; iv) a 7-min recovery period followed by a 300-s maximal effort ( $\left.\mathrm{TT}_{300}\right)$; and $\mathrm{v}$ ) a final 5-min recovery period. During recovery periods, athletes were asked to pedal at a power output of $1.2 \mathrm{~W}^{*} \mathrm{~kg}^{-1}$ body mass ( $>$ Fig. 1 ). The PPT was run in the simulation mode of the Cyclus2 ergometer. Thus the athletes were able to do it with their own CAD and in a seated or standing position. The gear ratio could be selected individually by electronically simulated shifting. In order to ensure that the athletes exerted themselves to the maximum, the testing instructor motivated them verbally as much as possible. The highest mean PO (including the zero values) for all time sections (10, 30, 60 and $300 \mathrm{~s}$ ) and the corresponding CAD were automatically calculated by the training software GoldenCheetah (www.goldencheetah.org; version 3.4). The level of recovery was not controlled, because a defined or even complete regeneration was neither necessary nor desirable.

\section{Simulated XCO race (Race)}

The Race was arranged specifically for the participants during the off-season on a slightly modified official XCO racetrack of the Union Cycliste Internationale (UCI) in (Albstadt, Germany). The modified lap with a $130 \mathrm{~m}$ ascent and about $2100 \mathrm{~m}$ length started at $750 \mathrm{~m}$ above sea level. The track was almost dry, there was neither rain nor any relevant wind, and the average air temperature was about $14^{\circ} \mathrm{C}$.

To account for age and gender differences in the given sample, races were performed separately for (a) female athletes and under-
- Table 1 Classification of XCO athletes (mean $\pm S D ; n=22$ ).

\begin{tabular}{|l|l|c|c|c|l|}
\hline & $\begin{array}{c}\text { U17 } \\
\text { [n] }\end{array}$ & $\begin{array}{c}\text { U19, U23, } \\
\text { Elite [n] }\end{array}$ & $\begin{array}{l}\text { Race a) - } \\
\text { 4 laps [n] }\end{array}$ & $\begin{array}{l}\text { Race b) - } \\
\text { 6 laps [n] }\end{array}$ & $\begin{array}{l}\text { Age } \\
\text { [years] }\end{array}$ \\
\hline Female & 2 & 4 & 6 & 0 & $16.8 \pm 1.8$ \\
\hline Male & 6 & 10 & 6 & 10 & $18.3 \pm 4.1$ \\
\hline Total & 8 & 14 & 12 & 10 & $17.9 \pm 3.7$ \\
\hline \multicolumn{6}{|l}{ U17/U19/U23: denotes athletes under 17/19/23 years of age. } \\
\hline
\end{tabular}

17 male athletes with 4 laps, and (b) male athletes over 17 years with 6 laps ( $\triangleright$ Table 1 ). This resulted in mean race duration of $43.8 \mathrm{~min}$ (37.7-55.7 min) for race (a) and 54.8 $\mathrm{min}$ (50.3-60.5 min) for race (b), respectively, which is approximately the recommended national race duration for juniors (50-70 min).

For each race, the athletes were positioned in two starting rows by the coaches according to their previous racing performance in order to avoid disadvantages due to the starting position and also encouraged to finish the race as fast as possible. In order to achieve the best possible comparability between laboratory and field measurements, the original cranks of the athletes' bikes were replaced by SRM training systems that continuously recorded PO, CAD, heart rate, and location/altitude. Additionally, the lap times were recorded manually with a stopwatch. As well as in the PPT, during the Race the highest mean PO (including the zero values) for all time sections (10,30,60, and $300 \mathrm{~s}$ ) and the corresponding CAD were automatically calculated by the training software GoldenCheetah (www.goldencheetah.org; version 3.4).

Immediately after the races, athletes were asked for possible interruptions of the race, such as falls or technical problems. Athletes with serious technical problems or severe health complaints during the races were excluded $(n=1)$.

\section{Statistical analysis}

Data were analysed with IBM SPSS Statistics version 25.0 (IBM Corp., Armonk, NY, USA). The distribution of data was checked 
- Table 2 Power Output and Cadence during Power Profile Test and XCO Race ( $\mathrm{n}=22)$.

\begin{tabular}{|c|c|c|c|c|c|c|c|c|c|c|c|}
\hline & \multirow{2}{*}{$\begin{array}{l}\text { PPT } \\
\text { Mean } \pm \text { SD }\end{array}$} & \multirow{2}{*}{$\begin{array}{l}\text { Race } \\
\text { Mean } \pm \text { SD }\end{array}$} & \multicolumn{2}{|l|}{ LoA } & \multicolumn{2}{|c|}{ LoA norm.n } & \multirow[t]{2}{*}{$\mathbf{r}$} & \multicolumn{4}{|c|}{ Difference } \\
\hline & & & Low & Up & Low & Up & & Abs. & $\begin{array}{l}\mathrm{Cl} \\
\text { low }\end{array}$ & $\mathrm{Cl}$ up & Norm. ${ }^{n}$ \\
\hline $\mathrm{PO} \mathrm{TT}_{10}[\mathrm{~W}]$ & $855 \pm 204$ & $781 \pm 157$ & -171 & 319 & -20 & 37 & $0.79^{*}$ & $74^{*}$ & 18 & 129 & $8 \%$ \\
\hline $\mathrm{PO} \mathrm{TT}_{30}[\mathrm{~W}]$ & $588 \pm 129$ & $549 \pm 119$ & -110 & 187 & -17 & 31 & $0.82^{*}$ & $39^{*}$ & 5 & 72 & $7 \%$ \\
\hline $\mathrm{PO} \mathrm{TT}_{60}[\mathrm{~W}]$ & $397 \pm 90$ & $460 \pm 107$ & -179 & 53 & -41 & 12 & $0.83^{*}$ & $-63^{*}$ & -89 & -37 & $-15 \%$ \\
\hline $\mathrm{PO} \mathrm{TT}_{300}[\mathrm{~W}]$ & $261 \pm 55$ & $293 \pm 54$ & -86 & 20 & -32 & 7 & $0.88^{*}$ & $-33^{*}$ & -45 & -21 & $-12 \%$ \\
\hline $\mathrm{CAD} \mathrm{TT}_{10}[\mathrm{rpm}]$ & $122 \pm 17$ & $111 \pm 12$ & -17 & 41 & -14 & 34 & $0.55^{*}$ & $12^{*}$ & 5 & 18 & $10 \%$ \\
\hline $\mathrm{CAD} \mathrm{TT}_{30}[\mathrm{rpm}]$ & $109 \pm 12$ & $100 \pm 8$ & -14 & 32 & -13 & 30 & 0.41 & $9^{*}$ & 4 & 14 & $8 \%$ \\
\hline $\mathrm{CAD} \mathrm{TT}_{60}[\mathrm{rpm}]$ & $110 \pm 11$ & $91 \pm 6$ & -3 & 42 & -2 & 40 & 0.26 & $20^{*}$ & 15 & 25 & $19 \%$ \\
\hline $\mathrm{CAD} \mathrm{TT}_{300}[\mathrm{rpm}]$ & $103 \pm 5$ & $84 \pm 5$ & 7 & 32 & 7 & 35 & 0.22 & $20^{*}$ & 17 & 22 & $21 \%$ \\
\hline
\end{tabular}

PPT, Power Profile Test; PO, cycling power output; CAD, cadence/pedalling frequency; $\mathrm{TT}_{10-300}$, time trials lasting 10-300 s (sprint/maximal effort); LoA, lower/upper $95 \%$ limits of agreement; ${ }^{n}$, normalized values (PPT-Race)/((PPT+ Race)/2) [\%]; r, Pearson correlation coefficient; * $=p<0.05 ;$ Abs., referring absolute values; $\mathrm{Cl}$, lower/upper $95 \%$ confidence interval of the difference.

using a Shapiro-Wilk test $(p>0.05)$ and a visual inspection of their histograms, normal Q-Q plots and box plots [31]. Descriptive results are presented as mean \pm standard deviation (SD). The PO and CAD data of PPT and Race were compared with paired sample t-tests. The level of significance was set at $\alpha=0.05$ (two-sided). Pearson's correlation coefficient was used to correlate data of PTT and Race as well as PO and CAD. The coefficient was interpreted using a categorization proposed by Hinkle et al. [32]: 0.9-1.0 very high; 0.7-0.9 high; 0.5-0.7 moderate; 0.3-0.5 low; 0.0-0.3 negligible correlation. Mean differences between PTT and Race and the corresponding $95 \%$ limits of agreement (LoA) were calculated as mean $\pm 1.96 *$ SD [33]. Additionally, the differences of PPT minus Race for each $P O$ and $C A D$, respectively, were divided by their mean to make them comparable (normalized difference).

\section{Results}

After the initial medical examination, 23 athletes who started in the current season in the national junior classes or the elite class were included in the study. Due to a technical defect, one athlete could not finish the XCO race $>$ Fig. 15 . Thus, in the end, 22 datasets were analysed ( $\triangleright$ Table $\mathbf{1}$ ).

Cycling power output and cadence data during the PPT and Race are summarized in > Table $\mathbf{2}$ for these 22 athletes. Differences in PO data were normally distributed except for $\mathrm{TT}_{30}\left(\mathrm{TT}_{10}=0.27\right.$, $\left.\mathrm{TT}_{30}=0.03, \mathrm{TT}_{60}=0.77, \mathrm{TT}_{300}=0.91\right)$. With regard to the $\mathrm{CAD}$ data, all differences were normally distributed $\left(\mathrm{TT}_{10}=0.13, \mathrm{TT}_{30}=0.74\right.$, $\left.\mathrm{TT}_{60}=0.97, \mathrm{TT}_{300}=0.51\right)$. Normalized differences of PO between the PPT and the Race are additionally presented in $>$ Fig. 2 for each of the assessed test durations, but CAD data is not shown in graphs.

Correlations of $\mathrm{PO}$ and $\mathrm{CAD}$ in total (aggregated data) were roughly moderate $(r=0.45 ; p<0.01)$ for the PPT as well as for the Race $(r=0.67 ; p<0.01)$ and are shown in $>$ Fig. 3 . The separately calculated correlations of PO and CAD for each of the TT were negligible ( $r<0.00$ to $0.27 ; p>0.05$ ). A low but statistically significant correlation $(r=0.42)$ of $\mathrm{PO}$ and $\mathrm{CAD}$ resulted only for $\mathrm{TT}_{300}$ during the race.

\section{Discussion}

In this study we compared cycling power output as well as cycling cadence during short-term time trials under laboratory and field conditions. Furthermore, the relationship between PO and CAD when cycling at these high intensities was examined.

We partially rejected the hypothesis that $\mathrm{PO}$ in laboratory conditions (PPT) and during an XCO race is not significantly different because we found higher PO during the PPT for the shorter efforts ( $\mathrm{TT}_{10}$ and $\mathrm{TT}_{30}$ ) but lower $\mathrm{PO}$ for $\mathrm{TT}_{60}$ and $\mathrm{TT}_{300}$ ( $\triangleright$ Table 2 ). Some differences between laboratory and field data were to be expected especially for the short sprints, because for tactical reasons short sprints are usually not performed at their maximum in competitions. However due to the tactical nature and requirements of XCO racing, it is more likely that a rider will give a maximal effort across each of the durations examined in the PPT than would be the case for road racing. This should give increased relevance to the comparison of maximum efforts in the laboratory with XCO race data compared to road racing.

Bertucci et al. [15] reported both higher $(+6 \%$, standing position) and lower ( $-4 \%$, seated) PO records during field-based sprint tests compared to stationary ergometer sprinting. Overall, these differences were slightly lower than in our measurements. However, in the study by Bertucci, measures were not performed in competition but in an isolated sprint test in a gymnasium with actual cycling from a static start. The authors attributed this difference in part to absent lateral oscillations of the bicycle ergometer and the associated reduction in the cyclists' ability to apply a perpendicular force to the pedals. They suggest that it would be desirable to use an ergometer that allows these lateral oscillations, such as the Cyclus2 ergometer used in our study. Interestingly, summarizing the feedback of our athletes, it can be stated that more natural cycling was experienced compared to previous testing with other ergometers. Unfortunately, we did not determine the extent of lateral oscillations that occurred. Quod et al. [13] compared laboratory $\mathrm{PO}$ and CAD data with road race data of ten male cyclists. Because cyclists competed in multiple races, the highest individual PO for each of the assessed durations was analysed. In contrast to our results, the authors reported higher PO (normalized differ- 

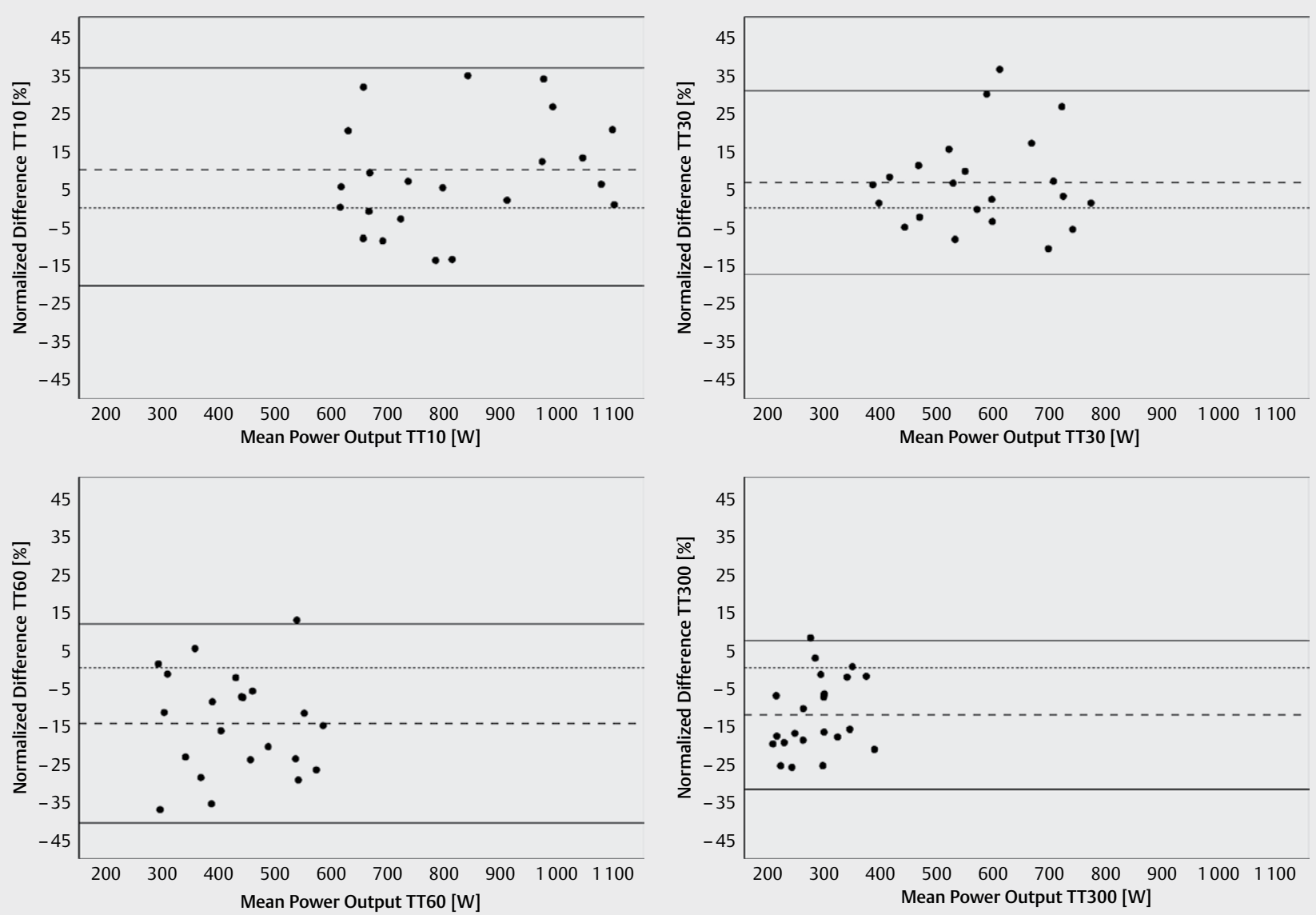

- Fig. 2 Normalized difference in power output between the Power Profile Test and the XCO race (PPT-Race); $n=22$ for each sprint $/ \mathrm{maximal}$ effort. The dotted line represents the exact match $(Y=0)$. The dashed line represents the mean bias and solid lines the $95 \%$ limits of agreement.

ences of 3-9\%) during races for the short sprints (5-30s) but found no PO differences for durations of $60-600$ s. These different results could be related to both the setting of the laboratory PPT and the type of competition (simulated XCO vs. multiple road races). The PPT in our study was designed to quantify cycling performance during XCO typical load periods of $10-300$ s [4, 5, 7]. Especially during very high intensity and maximal efforts (as demanded by the PPT), exercise capacity may also be limited by the amount of energy obtained from the anaerobic energy storage [34]. The GXT ahead of the PPT in our study could have affected the PO during the maximal efforts because athletes could not completely recuperate during the recovery periods, even though a substantial recovery in muscle function occurs within the first minutes after exercise termination [35]. By reason of increasing fatigue over time, the PO values of the sprints and maximal efforts are expected to be smaller than those seen without preloading. Despite all this, the PO values for $\mathrm{TT}_{10}$ and $\mathrm{TT}_{30}$ were higher during the PPT, although the highest $\mathrm{PO}$ over these time periods typically occurs at the start of the race and is predominated by anaerobic capacity. This indicates that it is possible to use the laboratory PPT to determine athletes' power-producing capacities during XCO races. The series of load peaks during the PPT in the absence of complete regeneration cor- responds to the requirements in XCO races but might be influenced by familiarisation and experience with the testing procedure. This test protocol in its entirety was validated with a good predictive power of the race performance in XCM [28] and XCO [9]. The authors reported that it explained $57 \%\left(\mathrm{TT}_{10}\right), 72 \%\left(\mathrm{TT}_{30}\right), 70 \%\left(\mathrm{TT}_{60}\right)$, and $74 \%\left(\mathrm{TT}_{300}\right)$ of the variance in $\mathrm{PO}$ during a $\mathrm{XCO}$ race [9].

As hypothesised, the self-selected CAD during the time trials differed remarkably and were statistically significant between the PPT and the Race ( $\triangleright$ Table 2). Quod et al. [13] reported considerably higher CAD (7-27 rpm) during laboratory measurements compared to actual cycling for similar time durations and PO values. Therefore they suggested that performing the laboratory test isokinetically and limiting the CAD to 95-100 rpm could be an alternative. However, due to the huge variation in self-selected CAD between athletes, a fixed CAD does not seem to be a better-suited test method in our opinion. As well as Nimmerichter and Williams [36], Bertucci et al. [15] also found higher CAD during ergometer tests compared to sprint tests at actual cycling locomotion, confirming the overall state of research. During prolonged cycling, a decrease in the self-selected CAD associated with neuromuscular fatigue has previously been reported $[19,37,38]$. Even if the race duration in XCO is significantly shorter than in road races, this could 


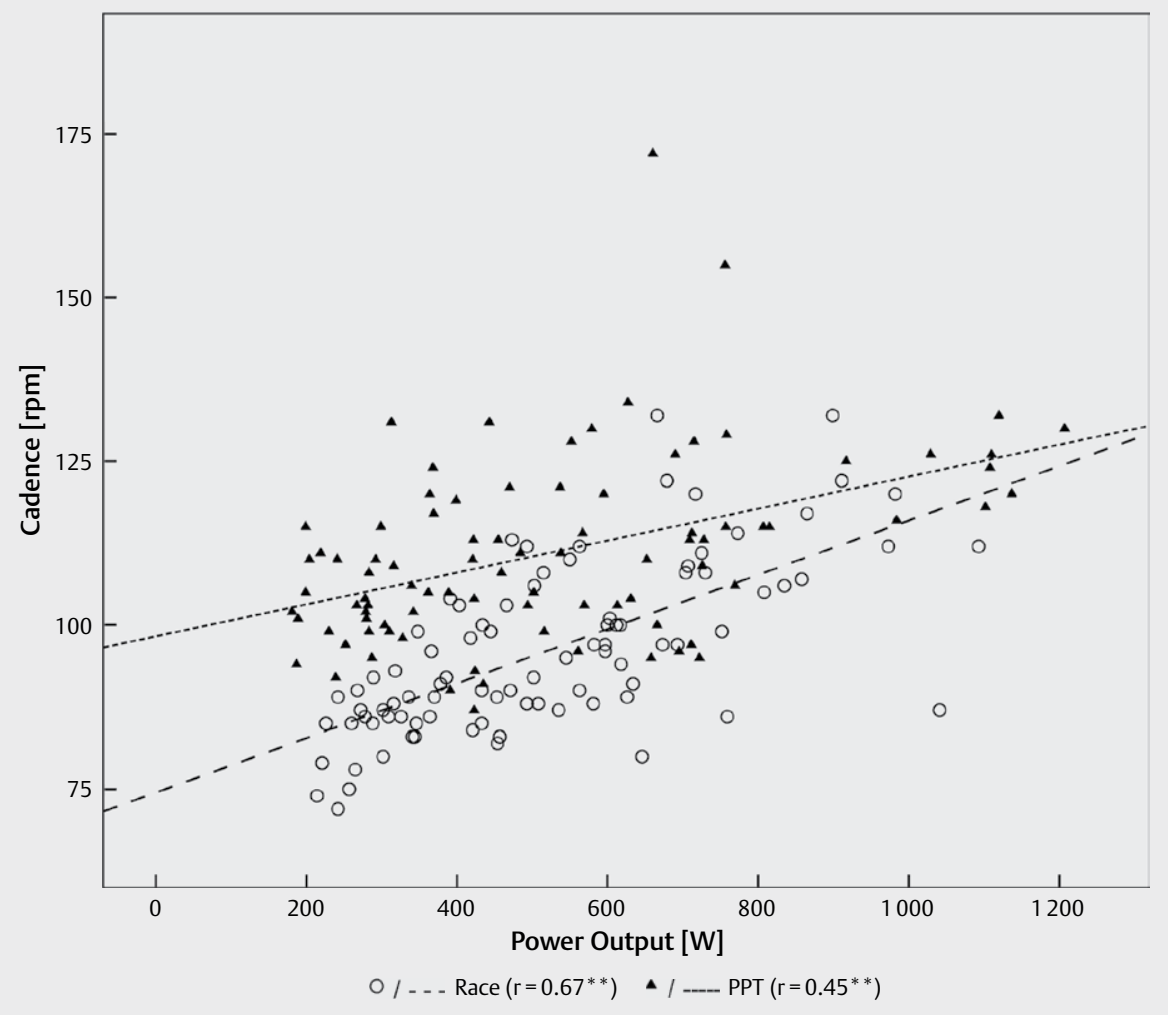

-Fig. 3 Correlation of aggregated power output and cadence; the lines represent the linear correlation for race data (Race) and the laboratory Power Profile Test (PPT); $n=88$ for each the Race and the PPT

play a role regarding the self-selected $C A D$. The partly remarkable lower CAD during the respective TT (Race) in our study could be due not only to economic aspects but also to the available gear ratio which is sometimes limited for (former) XCO typical 1x drivetrains. These and other external factors such as technical requirements or weather and track conditions remain as a difference between a real race and laboratory ergometer tests.

Most of the previous studies investigated the relationship between CAD and PO with distinctly lower PO values [18, 21, 23-26] than measured during short high-intensity intervals at cycling competitions [13-15]. Thus, the best (e.g. maximum power-producing) CAD cannot be reliably predicted for power outputs of more than $400 \mathrm{~W}$ [23]. The reported phenomenon that the most economical CAD increases with increasing workload could partly explain the fact that trained cyclists tend to adopt a higher CAD than untrained individuals [20]. Experienced cyclists may select higher CAD to minimize local muscle stress [16], even if the metabolic cost is actually higher. As hypothesised, our results showed an increase of CAD with increasing workload - or decreasing time duration for both the PPT (103-122 rpm) and the Race (84-111 rpm). The aggregated data ( $\triangleright$ Fig. 3 ) showed moderate and statistically significant correlations of PO and CAD during PPT $(r=0.45)$ and Race $(r=0.67)$. For similar time durations and PO values, Quod et al. [13] reported an increase of CAD with an increasing workload of 102119 during their laboratory PPT and 95-102 rpm during road races.
Considering the different underlying cycling disciplines with more constant conditions for road cycling, these results should be comparable. The variable gradients and technical requirements during an XCO race may have a much greater impact on the self-selected CAD than has been reported in road races $[39,40]$. The high selfselected $C A D$, especially under laboratory conditions, confirms the assumption that a higher $\mathrm{PO}$ also requires higher CAD. An athlete's individual CAD, however, differs considerably in some cases.

In this study, heart rate was not examined as exercise training is inspired by power-based training levels and high-intensity training zones to account for the short- and medium-term high-load events, such as XCO competitions. Therefore it is inappropriate to control the intensity of such short intervals (10-300s) using heart rate, especially under different environmental conditions if the possibility of power-controlled training exists.

In conclusion, the variability of $\mathrm{PO}$ and the confounding influence of tactics and external conditions during XCO races limit the explanatory power of comparisons between PO data collected from laboratory tests and XCO races. Nevertheless, the results $(r=0.79$ to 0.88 ) of this study indicate that it is possible to use the laboratory PPT to determine athletes' power-producing capacities during XCO races. However, the comparability of PO data from laboratory and field-based tests will always depend on the test method used. This must be taken into account when comparing results based on different study designs. 
The findings confirm the past research regarding the increase of CAD with increasing workload. However, it must be assumed that the "optimal cadence" in terms of maximum PO for such short sprints and maximal efforts lasting 10-300 s is higher than that reported in most previous studies. In future investigations and for training purposes, it should be taken into account that compared to the field, a higher CAD is likely to be used in laboratory settings to produce similar power outputs.

\section{Contributor's Statement}

None

\section{Acknowledgements}

We would like to thank all the volunteers who took part in this study that was supported by the Bundesinstitut für Sportwissenschaft (www.bisp.de) under grant [AZ 072041/16-17]. Furthermore we acknowledge support by Deutsche Forschungsgemeinschaft and Open Access Publishing Fund of University of Tübingen.

\section{Conflict of Interest}

The authors declare that they have no conflict of interest.

\section{References}

[1] Union Cycliste Internationale. UCI Cycling Regulations. Part 4. Mountain Bike.; Version on 01.01.2018. 1-70

[2] Baron R. Aerobic and anaerobic power characteristics of off-road cyclists. Med Sci Sports Exerc 2001; 33: 1387-1393

[3] Impellizzeri FM, Marcora SM. The physiology of mountain biking. Sports Med 2007; 37: 59-71. doi:10.2165/00007256-20073701000005

[4] Inoue A, Sa Filho AS, Mello FC et al. Relationship between anaerobic cycling tests and mountain bike cross-country performance. J Strength Cond Res 2012; 26: 1589-1593. doi:10.1519/JSC.0b013e318234eb89

[5] Stapelfeldt B, Schwirtz A, Schumacher YO et al. Workload demands in mountain bike racing. Int J Sports Med 2004; 25: 294-300. doi:10.1055/s-2004-819937

[6] Theobald U. Leistungsanforderungen und Trainingsmittel in der Radsportdisziplin Mountainbike Cross-Country. Leistungssport 2015; 1: 20-24

[7] Allen H, Coggan A. Training and Racing With A Power Meter. Boulder, CO: VeloPress. 2010

[8] Stevens C], Dascombe BJ. The reliability and validity of protocols for the assessment of endurance sports performance: an updated review. Meas Phys Educ Exerc Sci 2015; 19: 177-185. doi:10.1080/109136 7x.2015.1062381

[9] Schneeweiss P, Schellhorn P, Haigis D et al. Predictive Ability of a laboratory performance test in mountain bike cross-country Olympic athletes. Int J Sports Med 2019; 40: 397-403. doi:10.1055/a-0858-9900

[10] Coyle EF, Feltner ME, Kautz SA et al. Physiological and biomechanical factors associated with elite endurance cycling performance. Med Sci Sports Exerc 1991; 23: 93-107
[11] Palmer GS, Dennis SC, Noakes TD et al. Assessment of the reproducibility of performance testing on an air-braked cycle ergometer. Int J Sports Med 1996; 17: 293-298. doi:10.1055/s-2007-972849

[12] Smith MF, Davison RC, Balmer J et al. Reliability of mean power recorded during indoor and outdoor self-paced $40 \mathrm{~km}$ cycling time-trials. Int J Sports Med 2001; 22: 270-274. doi: $10.1055 / \mathrm{s}-2001-13813$

[13] Quod M], Martin DT, Martin JC et al. The power profile predicts road cycling MMP. Int J Sports Med 2010; 31: 397-401. doi:10.1055/s-0030-1247528

[14] Gardner AS, Martin JC, Martin DT et al. Maximal torque- and power-pedaling rate relationships for elite sprint cyclists in laboratory and field tests. Eur J Appl Physiol 2007; 101: 287-292. doi:10.1007| s00421-007-0498-4

[15] Bertucci W, Taiar R, Grappe F. Differences between sprint tests under laboratory and actual cycling conditions. J Sports Med Phys Fitness 2005; 45: 277-283

[16] Abbiss CR, Peiffer J], Laursen PB. Optimal cadence selection during cycling : Review article. Int SportMed J 2009; 10: 1-15

[17] Ansley L, Cangley P. Determinants of optimal cadence during cycling. Eur J Sport Sci 2009; 9: 61-85. doi:10.1080/17461390802684325

[18] Hansen EA, Andersen JL, Nielsen JS et al. Muscle fibre type, efficiency, and mechanical optima affect freely chosen pedal rate during cycling. Acta Physiol Scand 2002; 176: 185-194. doi:10.1046/j.1365-201X.2002.01032.x

[19] Vercruyssen F, Hausswirth C, Smith D et al. Effect of exercise duration on optimal pedaling rate choice in triathletes. Can J Appl Physiol 2001; 26: $44-54$

[20] Vercruyssen F, Brisswalter ]. Which factors determine the freely chosen cadence during submaximal cycling? I Sci Med Sport 2010; 13: 225-231. doi:10.1016/j.jsams.2008.12.631

[21] Hansen EA, Smith G. Factors affecting cadence choice during submaximal cycling and cadence influence on performance. Int J Sports Physiol Perform 2009; 4: 3-17

[22] Hill AV. The heat of shortening and the dynamic constants of muscle. Proceedings of the Royal Society of London Series B - Biological Sciences 1938; 126: 136-195. doi:10.1098/rspb.1938.0050

[23] Foss $\mathrm{O}$, Hallen J. The most economical cadence increases with increasing workload. Eur J Appl Physiol 2004; 92: 443-451. doi:10.1007/s00421-004-1175-5

[24] Foss O, Hallen J. Cadence and performance in elite cyclists. Eur ] Appl Physiol 2005; 93: 453-462. doi:10.1007/s00421-004-1226-y

[25] Coast JR, Cox RH, Welch HG. Optimal pedalling rate in prolonged bouts of cycle ergometry. Med Sci Sports Exerc 1986; 18: 225-230

[26] Boning D, Gonen Y, Maassen N. Relationship between work load, pedal frequency, and physical fitness. Int J Sports Med 1984; 5: 92-97. doi:10.1055/s-2008-1025887

[27] Harriss D], MacSween A, Atkinson G. Ethical standards in sport and exercise science research: 2020 update. Int J Sports Med 2019; 40: 813-817. doi:10.1055/a-1015-3123

[28] Ahrend MD, Schneeweiss P, Martus P et al. Predictive ability of a comprehensive incremental test in mountain bike marathon. BM] Open Sport Exerc Med 2018; 4: e000293. doi:10.1136/bmjsem-2017-000293

[29] Gardner AS, Stephens S, Martin DT et al. Accuracy of SRM and power tap power monitoring systems for bicycling. Med Sci Sports Exerc 2004; 36: 1252-1258. doi:10.1249/01.MSS.0000132380.21785.03

[30] Paton CD, Hopkins WG. Tests of cycling performance. Sports Med 2001; 31: 489-496. doi:10.2165/00007256-200131070-00004 
[31] Ghasemi A, ZahediasI S. Normality tests for statistical analysis: A guide for non-statisticians. Int J Endocrinol Metab 2012; 10: 486-489. doi:10.5812/ijem.3505

[32] Hinkle DE, Wiersma W, Jurs S. Applied Statistics for the Behavioral Sciences, International Edition. 5th ed. Belmont, CA: Wadsworth; 2009

[33] Bland JM, Altman DG. Statistical methods for assessing agreement between two methods of clinical measurement. Lancet 1986; 1 : 307-310. doi:10.1016/S0140-6736(86)90837-8

[34] Capelli C, Rittveger J, Bruseghini P et al. Maximal aerobic power and anaerobic capacity in cycling across the age spectrum in male master athletes. Eur J Appl Physiol 2016; 116: 1395-1410. doi:10.1007/ s00421-016-3396-9

[35] Froyd C, Millet GY, Noakes TD. The development of peripheral fatigue and short-term recovery during self-paced high-intensity exercise. J Physiol 2013; 591: 1339-1346. doi:10.1113/jphysiol.2012.245316
[36] Nimmerichter A, Williams CA. Comparison of power output during ergometer and track cycling in adolescent cyclists. J Strength Cond Res 2015; 29: 1049-1056. doi:10.1519/JSC.0000000000000723

[37] Jeukendrup AE, Moseley L, Mainwaring Gl et al. Exogenous carbohydrate oxidation during ultraendurance exercise. J Appl Physiol (1985) 2006; 100: 1134-1141. doi:10.1152/japplphysiol.00981.2004

[38] Lepers R, Hausswirth C, Maffiuletti N et al. Evidence of neuromuscular fatigue after prolonged cycling exercise. Med Sci Sports Exerc 2000; 32: $1880-1886$

[39] Lucia A, Hoyos J, Chicharro JL. Preferred pedalling cadence in professional cycling. Med Sci Sports Exerc 2001; 33: 1361-1366

[40] Vogt S, Schumacher YO, Roecker $K$ et al. Power output during the Tour de France. Int J Sports Med 2007; 28: 756-761. doi:10.1055/s-2007-964982 